$\mathrm{isid} / \mathrm{ms} / 2002 / 26$

October 10, 2002

http://www.isid.ac.in/ statmath/eprints

\title{
Non-uniform and uniform Berry-Esseen type bounds for stationary associated sequences
}

\author{
ISHA DEWAN \\ and \\ B.L.S. PRAKasa RaO
}

Indian Statistical Institute, Delhi Centre

7, SJSS Marg, New Delhi-110 016, India 


\title{
NON-UNIFORM AND UNIFORM BERRY-ESSEEN TYPE BOUNDS FOR STATIONARY ASSOCIATED SEQUENCES
}

\author{
Isha Dewan and B.L.S. Prakasa Rao \\ e-mail: isha@isid.ac.in , blsp@isid.ac.in \\ Indian Statistical Institute , 7, S.J.S. Sansanwal Marg \\ New Delhi 110016, INDIA
}

\begin{abstract}
Let $\left\{X_{n}, n \geq 1\right\}$ be a sequence of stationary associated random variables. We derive uniform and non-uniform Berry-Esseen type bounds for associated as well as functions of associated random variables. We discuss an application of these to kernel type density estimation for stationary associated random variables.
\end{abstract}

Key words : Berry-Esseen type bounds, stationary associated random variables, kernel type density estimation.

\section{Introduction}

Let $\left\{X_{i}, 1 \leq i \leq n\right\}$ be a set of stationary associated random variables, that is, for every pair of functions $h(\underline{x})$ and $g(\underline{x})$ from $R^{n}$ to $R$, which are nondecreasing componentwise,

$$
\operatorname{Cov}(h(\underline{X}), g(\underline{X})) \geq 0,
$$

whenever it is finite, where $\underline{X}=\left(X_{1}, X_{2}, \ldots, X_{n}\right)$.

Associated random variables are of considerable interest in reliability studies (cf. Esary, Proschan and Walkup (1967), Prakasa Rao and Dewan (2001), Roussas (1999)). A BerryEsseen type bound for the sum of stationary associated random variables were earlier discussed by Wood (1983) and Birkel (1988). The rate obtained by Wood is $O\left(n^{-1 / 5}\right)$, which is far from the optimal rate $O\left(n^{-1 / 2}\right)$ in the classical Berry-Esseen bound in the case of independent and identically distributed random variables (cf Feller (1977)). Birkel (1988), under certain set of conditions on $\left\{X_{n}\right\}$, obtained the rate of $O\left(n^{-1 / 2} \log ^{2} n\right)$. Even though the rate obtained by Birkel (1988) gives a vastly improved rate for the Berry-Esseen type bound under certain set of conditions, it is not clear how the constants involved in the bound depend on the moments of the random variables $\left\{X_{n}\right\}$. Dewan and Prakasa Rao (1997) obtained the bounds more explicitly in terms of moments of the sequence $\left\{X_{n}\right\}$ and bounds on the density function of the partial sums of independent copies of $X_{1}$. For completeness, we give the details in Section 2. We also obtain non-uniform Berry-Esseen type bounds in the Section 3 . In Section 4 we obtain uniform and non-uniform Berry-Esseen type bounds for functions of stationary associated random variables. 
Finally we discuss an application of these results to kernel type density estimation for stationary associated random variables .

\section{Uniform Berry-Esseen type bound for Stationary Associated Random Variables}

Theorem 2.1 : Let $\left\{X_{i}, 1 \leq i \leq n\right\}$ be a set of stationary associated random variables with $E\left[X_{1}\right]=0, \quad \operatorname{Var}\left[X_{1}\right]=\sigma^{2}>0 \quad$ and $\quad E\left[\left|X_{1}\right|^{3}\right]<\infty$. Suppose $X_{1}$ has an absolutely continuous distribution function. Let $S_{n}=\sum_{i=1}^{n} X_{i}$ and $s_{n}^{2}=\operatorname{Var}\left(S_{n}\right)$. Suppose $\frac{s_{n}^{2}}{n} \rightarrow \sigma^{2}$ as $\quad n \rightarrow \infty$. Let $F_{n}(x)$ be the distribution function of $\frac{S_{n}}{s_{n}}$ and $F_{n}^{*}($.$) be the distribution$ function of $\frac{\sum_{i=1}^{n} Z_{i}}{s_{n}}$ where $Z_{i}, 1 \leq i \leq n$ are i.i.d. with distribution function same as that of $X_{1}$. Let $m_{n}$ be a bound on the derivative of $F_{n}^{*}$. Then there exist absolute constants $B_{i}>0,1 \leq i \leq 3$, such that

$$
\begin{aligned}
\sup _{x}\left|F_{n}(x)-\Phi(x)\right| \leq & B_{1} \frac{d_{n}^{1 / 3} m_{n}^{2 / 3}}{s_{n}^{2 / 3}}+B_{2} \frac{E\left|X_{1}\right|^{3}}{\sqrt{n} \sigma^{3}} \\
& +B_{3}\left(\frac{s_{n}}{\sigma \sqrt{n}}-1\right)
\end{aligned}
$$

where $\Phi(x)$ is the distribution function of a standard normal random variable and

$$
d_{n}=\sum_{j=2}^{n}(n-j+1) \operatorname{Cov}\left(X_{1}, X_{j}\right) .
$$

Proof : Let $\psi_{n}(t)$ and $\psi_{n}^{*}(t)$ be the characteristic functions corresponding to $F_{n}($.$) and$ $F_{n}^{*}($.$) , respectively. Note that, for T>0$

$$
\begin{aligned}
\sup _{x}\left|F_{n}(x)-F_{n}^{*}(x)\right| \leq & \frac{1}{\pi} \int_{-T}^{T}\left|\frac{\psi_{n}(t)-\psi_{n}^{*}(t)}{t}\right| d t \\
& +24 \frac{m_{n}}{\pi T}
\end{aligned}
$$

by the basic smoothing inequality (cf. Feller (1997), Vol II, pp 512).

Applying Newman and Wright's (1981) inequality to the stationary associated set of random variables $\left\{X_{i}, 1 \leq i \leq n\right\}$, we have

$$
\begin{aligned}
\left|\psi_{n}(t)-\psi_{n}^{*}(t)\right| & \leq \sum_{1 \leq i<j \leq n} \frac{t^{2}}{s_{n}^{2}} \operatorname{Cov}\left(X_{i}, X_{j}\right) \\
& =\frac{t^{2}}{s_{n}^{2}} \sum_{i=1}^{n} \sum_{j=i+1}^{n} \operatorname{Cov}\left(X_{i}, X_{j}\right) \\
& =\frac{t^{2}}{s_{n}^{2}} \sum_{j=2}^{n}(n-j+1) \operatorname{Cov}\left(X_{1}, X_{j}\right) \\
& =\frac{t^{2}}{s_{n}^{2}} d_{n} .
\end{aligned}
$$


From (2.2) and (2.3), we get that

$$
\sup _{x}\left|F_{n}(x)-F_{n}^{*}(x)\right| \leq \frac{d_{n}}{\pi s_{n}^{2}} T^{2}+24 \frac{m_{n}}{\pi T} .
$$

Choose

$$
T=\left[\frac{24 m_{n} s_{n}^{2}}{d_{n}}\right]^{1 / 3}
$$

Then it follows that

$$
\sup _{x}\left|F_{n}(x)-F_{n}^{*}(x)\right| \leq \frac{2(24)^{2 / 3}}{\pi} \frac{d_{n}^{1 / 3} m_{n}^{2 / 3}}{s_{n}^{2 / 3}} .
$$

Applying the classical Berry-Esseen bound for the i.i.d. random variables $\left\{Z_{i}, 1 \leq i \leq n\right\}$ (cf. Feller (1977), Vol II, pp 515), we have

$$
\begin{aligned}
\sup _{x}\left|F_{n}^{*}(x)-\Phi\left(\frac{s_{n} x}{\sigma \sqrt{n}}\right)\right| & \leq C \frac{E\left|Z_{1}\right|^{3}}{\sqrt{n} \sigma^{3}} \\
& =C \frac{E\left|X_{1}\right|^{3}}{\sqrt{n} \sigma^{3}}
\end{aligned}
$$

where $C$ is an absolute constant such that $\frac{\sqrt{10}+3}{6 \sqrt{2 \pi}} \leq C \leq 0.7975$ (cf. Esseen (1956), Van Beek (1972)).

Further using a result from Petrov (1975, pp 114), we get that

$$
\sup _{x}\left|\Phi\left(\frac{s_{n} x}{\sigma \sqrt{n}}\right)-\Phi(x)\right| \leq \frac{\left(\frac{s_{n}}{\sigma \sqrt{n}}-1\right)}{\sqrt{2 \pi e}}
$$

since $s_{n}^{2} \geq n \sigma^{2}$.

Combining (2.6) to (2.8) we get our result.

Remark 2.2 : The bound given above can be made more explicit by bounding $m_{n}$ in (2.1). Suppose that the characteristic function of $X_{1}$ is absolutely integrable. Then, following Feller (1977, Vol II, pp 489), we have $\frac{S_{n}^{*}}{\sigma \sqrt{n}}$ has a density $g_{n}$ where $S_{n}^{*}=\sum_{i=1}^{n} Z_{i}$ and the density $g_{n}$ tends uniformly to the standard normal density. Since the standard normal density is bounded, it follows that $g_{n}$ is bounded and the bound is $\frac{2}{\sqrt{2 \pi}}$ for large $n$.

Since the density of $\frac{S_{n}^{*}}{\sigma \sqrt{n}}$ is $g_{n}(x)$, the density of $\frac{S_{n}^{*}}{s_{n}}$ is $\frac{s_{n}}{\sigma \sqrt{n}} g_{n}\left(\frac{s_{n} x}{\sigma \sqrt{n}}\right)$, which can be written in the form

$$
\left(\frac{s_{n}}{\sigma \sqrt{n}}-1\right) g_{n}\left(\frac{s_{n} x}{\sigma \sqrt{n}}\right)+g_{n}\left(\frac{s_{n} x}{\sigma \sqrt{n}}\right) .
$$

Hence

$$
\begin{aligned}
m_{n} & \leq\left(\frac{s_{n}}{\sigma \sqrt{n}}-1\right) \sup _{x} g_{n}\left(\frac{s_{n} x}{\sigma \sqrt{n}}\right)+\sup _{x} g_{n}\left(\frac{s_{n} x}{\sigma \sqrt{n}}\right) \\
& \leq \frac{2}{\sqrt{2 \pi}}\left(\frac{s_{n}}{\sigma \sqrt{n}}-1\right)+\frac{2}{\sqrt{2 \pi}}
\end{aligned}
$$

for large $n$. 


\section{Non -Uniform Berry-Esseen type bounds}

In this section we find non-uniform Berry-Esseen type bounds following the techniques of Petrov (1975) and Hall (1982). Note that $C$ is a generic positive constant.

\subsection{Petrov's Approach (Stationary Associated Sequences )}

Let $F_{1}$ and $F_{2}$ be two distribution functions. If the distributions have moments of order $p>0$, then Petrov (1975, pp 120) proved that

$$
\left|F_{1}(x)-F_{2}(x)\right| \leq \frac{\beta}{|x|^{p}}, x \neq 0
$$

where $\beta=\max \left(\beta_{1}, \beta_{2}\right), \beta_{k}=\int_{-\infty}^{\infty}|x|^{p} d F_{k}(x)$. Furthermore

$$
\left|F_{1}(x)-F_{2}(x)\right| \leq\left\{\frac{(1+\beta)^{s} \Delta_{1}^{r}}{\left.1+|x|^{p}\right)^{s}}\right\}^{1 /(r+s)},
$$

for all $x$, for $r>0, s>0, \Delta_{1}=\sup _{x}\left|F_{1}(x)-F_{2}(x)\right|$.

Let $F(x)$ be an arbitrary distribution function and $\Phi(x)$ the standard normal distribution function. Let

$$
\Delta=\sup _{x}|F(x)-\Phi(x)|
$$

Further suppose that

$$
\int_{-\infty}^{\infty}|x|^{p} d F(x)<\infty
$$

for some $p>0$. The following theorem is due to Petrov (1975, pp 121).

Theorem 3.1 : Suppose that $0<\Delta \leq e^{-1 / 2}$ and the inequality (3.3) is satisfied. Then

$$
|F(x)-\Phi(x)| \leq \frac{c(p) \Delta(\log (1 / \Delta))^{p / 2}+\lambda_{p}}{1+|x|^{p}}
$$

for all $x$, where $c(p)$ is a positive constant depending only on $p$, and

$$
\lambda_{p}=\left.\left|\int_{-\infty}^{\infty}\right| x\right|^{p} d F(x)-\int_{-\infty}^{\infty}|x|^{p} d \Phi(x) \mid
$$

Let $F_{n}(x)$ and $F_{n}^{*}(x)$ be the distribution functions of $\frac{S_{n}}{s_{n}}$ and $\frac{S_{n}^{*}}{s_{n}}$, respectively, where $S_{n}=$ $\sum_{i=1}^{n} X_{i}$ and $S_{n}^{*}=\sum_{i=1}^{n} Z_{i}$ are as defined in Section 2. Then $\operatorname{Var} \frac{S_{n}}{s_{n}}=1$ and $\operatorname{Var} \frac{S_{n}^{*}}{s_{n}} \leq 1$ by the associative property of the random variables $X_{1}, \ldots, X_{n}$. Hence

$$
\left|F_{n}(x)-F_{n}^{*}(x)\right| \leq\left\{\frac{2 \Delta_{1 n}}{1+x^{2}}\right\}^{1 / 2}
$$

from (3.1) by choosing $p=2, r=1$ and $s=1$ where

$$
\Delta_{1 n}=\sup _{x}\left|F_{n}(x)-F_{n}^{*}(x)\right| \leq C \frac{d_{n}{ }^{1 / 3} m_{n}{ }^{2 / 3}}{s_{n}{ }^{2 / 3}},
$$


from (2.6). Further there exists an absolute constant $c(2)>0$ such that

$$
\left|F_{n}^{*}(x)-\Phi(x)\right| \leq \frac{c(2) \Delta_{2 n} \log \left(1 / \Delta_{2 n}\right)+\lambda_{2 n}}{1+x^{2}},
$$

where

$$
\begin{aligned}
\Delta_{2 n} & =\sup _{x}\left|F_{n}^{*}(x)-\Phi(x)\right|, \\
\lambda_{2 n} & =\left|\int_{-\infty}^{\infty} x^{2} d F_{n}^{*}(x)-1\right| \\
& =\left|\operatorname{Var}\left(S_{n}^{*} / s_{n}\right)-1\right| \\
& =\left|n \sigma^{2} / s_{n}^{2}-1\right| .
\end{aligned}
$$

Note that $\Delta_{2 n}$ is the Berry-Esseen bound obtained from (2.7) and (2.8).

Hence we have the following Theorem.

Theorem 3.2 : Let $\left\{X_{n}, n \geq 1\right\}$ be a sequence of stationary associated random variables satisfying the conditions of Theorem 2.1. Suppose that $0<\Delta_{2 n}<e^{-1 / 2}$. Then there exist absolute positive constants $c$ and $c(2)$ such that

$$
\left|F_{n}(x)-\Phi(x)\right| \leq c\left\{\frac{2 \Delta_{1 n}}{1+x^{2}}\right\}^{1 / 2}+\frac{c(2) \Delta_{2 n} \log \left(1 / \Delta_{2 n}\right)+\lambda_{2 n}}{1+x^{2}},
$$

for all $x$.

\subsection{Hall's Approach (General Case)}

Following Hall (1982, pp 33), we now obtain another non-uniform bound between the distribution function of $\sum_{i=1}^{n} X_{i}$ for a random vector $\left(X_{1}, \ldots, X_{n}\right)$ and the distribution function of $\sum_{i=1}^{n} Z_{i}$, where $Z_{i}, 1 \leq i \leq n$ are independent random variables and $Z_{i}$ has the same distribution as $X_{i}, 1 \leq i \leq n$.

Theorem 3.3: Let $F(x)$ be a non-decreasing function and $G(x)$ be a function of bounded variation on $\mathrm{R}$ and suppose that $F(-\infty)=G(-\infty)$ and $F(\infty)=G(\infty)$. Assume that

$$
\int_{-\infty}^{\infty} x^{2}|d\{F(x)-G(x)\}|<\infty
$$

and

$$
\chi(t)=\int_{-\infty}^{\infty} \exp (i t x) d\left[x^{2}\{F(x)-G(x)\}\right]
$$

Further suppose that

$$
\sup _{-\infty<x<\infty}\left(1+x^{2}\right)\left|G^{\prime}(x)\right| \leq k<\infty,
$$

where $G^{\prime}(x)$ is the derivative of $G(x)$. Let $f(t)$ and $g(t)$ denote the Fourier -Stieltjes transforms of $F$ and $G$, respectively. Then

$$
\sup _{x}\left(1+x^{2}\right)|F(x)-G(x)| \leq C\left\{\int_{0}^{T} \frac{|f(t)-g(t)|}{t} d t+\int_{0}^{T} \frac{|\chi(t)|}{t} d t+\frac{k}{T}\right\}
$$


for all $T \geq 1$ where $C$ is a universal positive constant (cf. Hall (1982)).

Let $H(x)=F(x)-G(x)$. Then

$$
\begin{aligned}
\chi(t) & =\int_{-\infty}^{\infty} \exp (i t x) d\left(x^{2} H(x)\right) \\
& =\int_{-\infty}^{\infty} \exp (i t x)\left\{x^{2} d H(x)+2 x H(x) d x\right\} \\
& =\int_{-\infty}^{\infty} \exp (i t x) x^{2} d H(x)+2 \int_{-\infty}^{\infty} \exp (i t x) x H(x) d x .
\end{aligned}
$$

Let

$$
\begin{aligned}
\gamma(t) & =f(t)-g(t) \\
& =\int_{-\infty}^{\infty} \exp (i t x) d(F(x)-G(x)) \\
& =\int_{-\infty}^{\infty} \exp (i t x) d H(x),
\end{aligned}
$$

Then

$$
\gamma^{\prime}(t)=\int_{-\infty}^{\infty} i x \exp (i t x) d H(x)
$$

and

$$
\gamma^{\prime \prime}(t)=i^{2} \int_{-\infty}^{\infty} x^{2} \exp (i t x) d H(x)
$$

Hence

$$
\begin{aligned}
\chi(t) & =-\gamma^{\prime \prime}(t)-2 i \frac{d}{d t}\left\{\int_{-\infty}^{\infty} \exp (i t x) H(x) d x\right\} \\
& =-\gamma^{\prime \prime}(t)+2 \frac{d}{d t}\left\{\frac{\gamma(t)}{t}\right\} \\
& =-\gamma^{\prime \prime}(t)+2\left(\frac{\gamma^{\prime}(t)}{t}-\frac{\gamma(t)}{t^{2}}\right) .
\end{aligned}
$$

\section{Bivariate Case}

Let $(X, Y)$ be a bivariate random vector and let $F(x)=P(X+Y \leq x)$. Let $(\tilde{X}, \tilde{Y})$ be another random vector with distribution of $\tilde{X}$ and $\tilde{Y}$ same as that of $X$ and $Y$, but $\tilde{X}$ and $\tilde{Y}$ are independent. Let $G(x)=P(\tilde{X}+\tilde{Y} \leq x)$. Note that $F$ is nondecreasing and $G$ is nondecreasing and hence a function of bounded variation. Furthermore $F(-\infty)=G(-\infty)=0$ and $F(\infty)=G(\infty)=1$. Assume that the condition (3.13) holds for the function $G($.$) . Then$

$$
\begin{aligned}
\gamma(t) & =\int_{-\infty}^{\infty} \exp (i t x) d(F(x)-G(x)) \\
& =E[\exp (i t(X+Y))]-E[\exp (i t X)] E[\exp (i t Y)] \\
& =\operatorname{Cov}(\exp (i t X), \exp (i t Y)) \\
& =\iint\left(i^{2} t^{2}\right) \exp (i t(x+y)) H(x, y) d x d y
\end{aligned}
$$


where

$$
H_{X Y}(x, y) \equiv H(x, y)=P[X<x, Y<y]-P[X<x] P[Y<y]
$$

Then

$$
\begin{aligned}
\gamma^{\prime}(t) & =\operatorname{Cov}(i X \exp (i t X), \exp (i t Y))+\operatorname{Cov}(\exp (i t X), i Y \exp (i t Y)) \\
& =-\iint t(2+x i t+y i t) \exp (i t(x+y)) H(x, y) d x d y
\end{aligned}
$$

and

$$
\begin{aligned}
\gamma^{\prime \prime}(t)= & -\operatorname{Cov}\left(X^{2} \exp (i t X), \exp (i t Y)\right)-\operatorname{Cov}\left(\exp (i t X), Y^{2} \exp (i t Y)\right) \\
& -2 \operatorname{Cov}(X \exp (i t X), Y \exp (i t Y)) \\
= & -\iint\left(2+4 i t(x+y)-(x+y)^{2} t^{2}\right) \exp (i t(x+y)) H(x, y) d x d y
\end{aligned}
$$

Hence, after simplification,

$$
\chi(t)=\iint \exp (i t(x+y))\left[2 i t(x+y)-(x+y)^{2} t^{2}\right] H(x, y) d x d y .
$$

Therefore

$$
|\chi(t)| \leq|t| A_{1}+|t|^{2} A_{2},
$$

And from (3.20), we get that

$$
|\gamma(t)| \leq t^{2} A_{0} .
$$

Hence, by using (3.24) and (3.25) in (3.14), it follows that there exists an absolute constant $C>0$ such that

$$
\sup _{x}\left(1+x^{2}\right)|P[X+Y \leq x]-P[\tilde{X}+\tilde{Y} \leq x]| \leq C\left[\left(A_{0}+2 A_{1}+A_{2}\right) T^{2}+K / T\right],
$$

where

$$
\begin{aligned}
& A_{0}=\int_{-\infty}^{\infty} \int_{-\infty}^{\infty}|H(x, y)| d x d y \\
& A_{1}=\int_{-\infty}^{\infty} \int_{-\infty}^{\infty}|x+y||H(x, y)| d x d y \\
& A_{2}=\int_{-\infty}^{\infty} \int_{-\infty}^{\infty}(x+y)^{2}|H(x, y)| d x d y
\end{aligned}
$$

Choosing $T=\left(A_{0}+2 A_{1}+A_{2}\right)^{-1 / 3}$, we get

$$
\sup _{x}\left(1+x^{2}\right)|P[X+Y \leq x]-P[\tilde{X}+\tilde{Y} \leq x]| \leq C K^{\frac{2}{3}}\left(A_{0}+2 A_{1}+A_{2}\right)^{\frac{1}{3}} .
$$

\section{Trivariate Case}

Let $X, Y, Z$ be random variables and suppose $F(x)$ and $G(x)$ are defined as earlier for the trivariate case. Suppose the condition (3.13) holds for the function $G(x)$. 
For more than two variables, the function $\gamma(t)$ is expressed in terms of the cumulants which are defined as below.

Definition : (Block and Fang (1988)) The rth-order joint cumulant of $\left(X_{1}, \ldots, X_{r}\right)$, denoted by $\operatorname{cum}\left(X_{1}, \ldots, X_{r}\right)$, is defined by

$$
\operatorname{cum}\left(X_{1}, \ldots, X_{r}\right)=\sum(-1)^{p-1}(p-1) !\left(E \prod_{j \in v_{1}} X_{j}\right) \ldots\left(E \prod_{j \in v_{p}} X_{j}\right)
$$

where summation extends over all partitions $\left(v_{1}, \ldots, v_{p}\right), p=1,2 \ldots, r$, of $(1, \ldots, r)$.

For real valued functions $f_{i}, i=1, \ldots, r$, assume that $E\left|f_{i}\left(X_{i}\right)\right|^{r}<\infty$. Then Dewan and Prakasa Rao (2000) proved the following result.

Lemma 3.4: If $E\left|f_{i}\left(X_{i}\right)\right|^{m}<\infty$, then

$$
\begin{aligned}
E & {\left[f_{1}\left(X_{1}\right) \ldots f_{m}\left(X_{m}\right)\right]-\prod_{i=1}^{m} E\left[f_{i}\left(X_{i}\right)\right] } \\
& =\sum \operatorname{cum}\left(f_{k}\left(X_{k}\right), k \in v_{1}\right) \ldots \operatorname{cum}\left(f_{k}\left(X_{k}\right), k \in v_{p}\right),
\end{aligned}
$$

where $\sum$ extends over all partitions $\left(v_{1}, \ldots, v_{p}\right), p=1, \ldots, m-1$, of $\{1, \ldots, m\}$.

In particular, for $m=3$, we have

$$
\begin{aligned}
E[ & \left.f_{1}\left(X_{1}\right) f_{2}\left(X_{2}\right) f_{3}\left(X_{3}\right)\right]-\prod_{i=1}^{3} E\left[f_{i}\left(X_{i}\right)\right] \\
= & \operatorname{cum}\left(f_{1}\left(X_{1}\right), f_{2}\left(X_{2}\right), f_{3}\left(X_{3}\right)\right)+E\left[f_{1}\left(X_{1}\right)\right] \operatorname{cum}\left(f_{3}\left(X_{3}\right), f_{2}\left(X_{2}\right)\right) \\
& \quad+E\left[f_{2}\left(X_{2}\right)\right] \operatorname{cum}\left(f_{1}\left(X_{1}\right), f_{3}\left(X_{3}\right)\right)+E\left[f_{3}\left(X_{3}\right)\right] \operatorname{cum}\left(f_{1}\left(X_{1}\right), f_{2}\left(X_{2}\right)\right) .
\end{aligned}
$$

Cumulants of functions can be expressed as follows. For details see Dewan and Prakasa Rao (2000).

Theorem 3.5 : If $E\left|f_{i}\left(X_{i}\right)\right|^{r}<\infty$ and $f_{i}$ is differentiable for $i=1, \ldots, r$, then

$$
\begin{aligned}
\operatorname{cum} & \left(f_{1}\left(X_{1}\right), \ldots, f_{r}\left(X_{r}\right)\right) \\
& =\int_{-\infty}^{\infty} \ldots \int_{-\infty}^{\infty} \prod_{i=1}^{r} f_{i}^{\prime}\left(x_{i}\right) \operatorname{cum}\left(\chi_{X_{1}}\left(x_{1}\right), \ldots, \chi_{X_{r}}\left(x_{r}\right)\right) d x_{1} \ldots d x_{r}
\end{aligned}
$$

where

$$
\chi_{X_{i}}\left(x_{i}\right)= \begin{cases}1 & \text { if } X_{i} \geq x_{i} \\ 0 & \text { otherwise }\end{cases}
$$


Observe that

$$
\begin{aligned}
\gamma(t) & =E(\exp (i t(X+Y+Z)))-E(\exp (i t X)) E(\exp (i t Y)) E(\exp (i t Z)) \\
& =\operatorname{Cum}\left(e^{i t X}, e^{i t Y}, e^{i t Z}\right)+E\left(e^{i t X}\right) \operatorname{Cov}\left(e^{i t Y}, e^{i t Z}\right) \\
& +E\left(e^{i t Y}\right) \operatorname{Cov}\left(e^{i t X}, e^{i t Z}\right)+E\left(e^{i t Z}\right) \operatorname{Cov}\left(e^{i t X}, e^{i t Y}\right) .
\end{aligned}
$$

Therefore, by Theorem 3.4,

$$
\begin{aligned}
|\gamma(t)| & \leq \int_{-\infty}^{\infty} \int_{-\infty}^{\infty} \int_{-\infty}^{\infty}\left|\operatorname{Cum}\left(\chi_{X}(x), \chi_{Y}(y), \chi_{Z}(z)\right)\right| d x d y d z \\
& +t^{2} \int_{-\infty}^{\infty} \int_{-\infty}^{\infty}\left|H_{X Y}(x, y)\right| d x d y+t^{2} \int_{-\infty}^{\infty} \int_{-\infty}^{\infty}\left|H_{Y Z}(y, z)\right| d y d z \\
& +t^{2} \int_{-\infty}^{\infty} \int_{-\infty}^{\infty}\left|H_{Z X}(z, x)\right| d x d z
\end{aligned}
$$

Furthermore

$$
\begin{aligned}
\gamma^{\prime}(t) & =\operatorname{Cum}\left(i X e^{i t X}, e^{i t Y}, e^{i t Z}\right)+E\left(i X e^{i t X}\right) \operatorname{Cov}\left(e^{i t Y}, e^{i t Z}\right) \\
& +E\left(e^{i t Y}\right) \operatorname{Cov}\left(i X e^{i t X}, e^{i t Z}\right)+E\left(e^{i t Z}\right) \operatorname{Cov}\left(i X e^{i t X}, e^{i t Y}\right) \\
& +\operatorname{Cum}\left(e^{i t X}, i Y e^{i t Y}, e^{i t Z}\right)+E\left(e^{i t X}\right) \operatorname{Cov}\left(i Y e^{i t Y}, e^{i t Z}\right) \\
& +E\left(i Y e^{i t Y}\right) \operatorname{Cov}\left(e^{i t X}, e^{i t Z}\right)+E\left(e^{i t Z}\right) \operatorname{Cov}\left(e^{i t X}, i Y e^{i t Y}\right) \\
& +\operatorname{Cum}\left(e^{i t X}, e^{i t Y}, i Z e^{i t Z}\right)+E\left(e^{i t X}\right) \operatorname{Cov}\left(e^{i t Y}, i Z e^{i t Z}\right) \\
& +E\left(e^{i t Y}\right) \operatorname{Cov}\left(e^{i t X}, i Z e^{i t Z}\right)+E\left(i Z e^{i t Z}\right) \operatorname{Cov}\left(e^{i t X}, e^{i t Y}\right) .
\end{aligned}
$$

Similarly, we have

$$
\begin{aligned}
-\gamma^{\prime \prime}(t) & =\operatorname{Cum}\left(X^{2} e^{i t X}, e^{i t Y}, e^{i t Z}\right)+E\left(X^{2} e^{i t X}\right) \operatorname{Cov}\left(e^{i t Y}, e^{i t Z}\right) \\
& +E\left(e^{i t Y}\right) \operatorname{Cov}\left(X^{2} e^{i t X}, e^{i t Z}\right)+E\left(e^{i t Z}\right) \operatorname{Cov}\left(X^{2} e^{i t X}, e^{i t Y}\right) \\
& +\operatorname{Cum}\left(e^{i t X}, Y^{2} e^{i t Y}, e^{i t Z}\right)+E\left(e^{i t X}\right) \operatorname{Cov}\left(Y^{2} e^{i t Y}, e^{i t Z}\right) \\
& +E\left(Y^{2} e^{i t Y}\right) \operatorname{Cov}\left(e^{i t X}, e^{i t Z}\right)+E\left(e^{i t Z}\right) \operatorname{Cov}\left(e^{i t X}, Y^{2} e^{i t Y}\right) \\
& +\operatorname{Cum}\left(e^{i t X}, e^{i t Y}, Z^{2} e^{i t Z}\right)+E\left(e^{i t X}\right) \operatorname{Cov}\left(e^{i t Y}, Z^{2} e^{i t Z}\right) \\
& +E\left(e^{i t Y}\right) \operatorname{Cov}\left(e^{i t X}, Z^{2} e^{i t Z}\right)+E\left(Z^{2} e^{i t Z}\right) \operatorname{Cov}\left(e^{i t X}, e^{i t Y}\right) \\
& +2 \operatorname{Cum}\left(X e^{i t X}, Y e^{i t Y}, e^{i t Z}\right)+2 E\left(X e^{i t X}\right) \operatorname{Cov}\left(Y e^{i t Y}, e^{i t Z}\right) \\
& +2 E\left(Y e^{i t Y}\right) \operatorname{Cov}\left(X e^{i t X}, e^{i t Z}\right)+2 E\left(e^{i t Z}\right) \operatorname{Cov}\left(X e^{i t X}, Y e^{i t Y}\right) \\
& +2 \operatorname{Cum}\left(e^{i t X}, Y e^{i t Y}, Z e^{i t Z}\right)+2 E\left(e^{i t X}\right) \operatorname{Cov}\left(Y e^{i t Y}, Z e^{i t Z}\right) \\
& +2 E\left(Y e^{i t Y}\right) \operatorname{Cov}\left(e^{i t X}, Z e^{i t Z}\right)+2 E\left(Z e^{i t Z}\right) \operatorname{Cov}\left(e^{i t X}, Y e^{i t Y}\right) \\
& +2 \operatorname{Cum}\left(X e^{i t X}, e^{i t Y}, Z e^{i t Z}\right)+2 E\left(X e^{i t X}\right) \operatorname{Cov}\left(e^{i t Y}, Z e^{i t Z}\right) \\
& +2 E\left(e^{i t Y}\right) \operatorname{Cov}\left(X e^{i t X}, Z e^{i t Z}\right)+2 E\left(Z e^{i t Z}\right) \operatorname{Cov}\left(X e^{i t X}, e^{i t Y}\right) .
\end{aligned}
$$

Then, it is easy to see that

$$
|\chi(t)| \leq 2 t \int_{-\infty}^{\infty} \int_{-\infty}^{\infty} \int_{-\infty}^{\infty}\left|\operatorname{Cum}\left(\chi_{X}(x), \chi_{Y}(y), \chi_{Z}(z)\right)\right| d x d y d z
$$




$$
\begin{aligned}
& +4 t^{2} \int_{-\infty}^{\infty} \int_{-\infty}^{\infty} \int_{-\infty}^{\infty}|x+y+z|\left|\operatorname{Cum}\left(\chi_{X}(x), \chi_{Y}(y), \chi_{Z}(z)\right)\right| d x d y d z \\
& +t^{3} \int_{-\infty}^{\infty} \int_{-\infty}^{\infty} \int_{-\infty}^{\infty}(x+y+z)^{2}\left|\operatorname{Cum}\left(\chi_{X}(x), \chi_{Y}(y), \chi_{Z}(z)\right)\right| d x d y d z \\
& +\left(2 t+t^{2}\right)\left[\int_{-\infty}^{\infty} \int_{-\infty}^{\infty}\left|H_{X Y}(x, y)\right| d x d y\right. \\
& \left.+\int_{-\infty}^{\infty} \int_{-\infty}^{\infty}\left|H_{Y Z}(y, z)\right| d y d z+\int_{-\infty}^{\infty} \int_{-\infty}^{\infty}\left|H_{X Z}(x, z)\right| d x d z\right] \\
& +2\left(t+t^{2}\right)\left[\int_{-\infty}^{\infty} \int_{-\infty}^{\infty}|x+y|\left|H_{X Y}(x, y)\right| d x d y\right. \\
& \left.+\int_{-\infty}^{\infty} \int_{-\infty}^{\infty}|y+z|\left|H_{Y Z}(y, z)\right| d y d z+\int_{-\infty}^{\infty} \int_{-\infty}^{\infty}|x+z|\left|H_{X Z}(x, z)\right| d x d z\right] \\
& +t^{2}\left[\int_{-\infty}^{\infty} \int_{-\infty}^{\infty}(x+y)^{2}\left|H_{X Y}(x, y)\right| d x d y\right. \\
& \left.+\int_{-\infty}^{\infty} \int_{-\infty}^{\infty}(y+z)^{2}\left|H_{Y Z}(y, z)\right| d y d z+\int_{-\infty}^{\infty} \int_{-\infty}^{\infty}(x+z)^{2}\left|H_{X Z}(x, z)\right| d x d z\right]
\end{aligned}
$$

where $H_{Y Z}$ and $H_{X Z}$ are defined analogous to (3.21). Let

$$
A_{X Y}^{r}=\int_{-\infty}^{\infty} \int_{-\infty}^{\infty}|x+y|^{r}\left|\operatorname{Cum}\left(\chi_{X}(x), \chi_{Y}(y)\right)\right| d x d y
$$

and

$$
A_{X Y Z}^{r}=\int_{-\infty}^{\infty} \int_{-\infty}^{\infty} \int_{-\infty}^{\infty}|x+y+z|^{r}\left|\operatorname{Cum}\left(\chi_{X}(x), \chi_{Y}(y), \chi_{Z}(z)\right)\right| d x d y d z
$$

for $r=0,1,2 . A_{Y Z}^{r}$ and $A_{X Z}^{r}$ are defined analogous to (3.39). Then, from (3.14), (3.34), (3.39) and (3.40), we get that there exists an absolute constant $C>0$ such that

$$
\begin{aligned}
& \sup _{x}\left(1+x^{2}\right)|P[X+Y+Z \leq x]-G(x)| \\
& \quad \leq C\left\{\left[\max _{r=0,1,2}\left(A_{X Y Z}^{r}, A_{X Y}^{r}, A_{Y Z}^{r}, A_{X Z}^{r}\right)\right] T^{3}+\frac{K}{T}\right\}
\end{aligned}
$$

for all $T>0$ so that

$$
\begin{aligned}
& \sup _{x}\left(1+x^{2}\right)|P[X+Y+Z \leq x]-G(x)| \\
& \quad \leq C K^{\frac{3}{4}}\left\{\max _{r=0,1,2}\left(A_{X Y Z}^{r}, A_{X Y}^{r}, A_{Y Z}^{r}, A_{X Z}^{r}\right)\right\}^{\frac{1}{4}}
\end{aligned}
$$

\section{n-variate case}

For the general case we have the following result. We omit the proof.

Theorem 3.6 : Let $\left(X_{1}, \ldots, X_{n}\right)$ be a random vector with $\tilde{F}_{n}(x)=$ $P\left(X_{1}+\ldots+X_{n} \leq x\right)$. Let $Z_{i}$ have the same distribution as $X_{i}, 1 \leq i \leq n$ and suppose that $Z_{i}, 1 \leq i \leq n$ are independent . Suppose $G_{n}(x), g_{n}(x)$ are respectively, the distribution function and the density function of $\sum_{i=1}^{n} Z_{i}$. Further suppose that

$$
\sup _{-\infty<x<\infty}\left(1+x^{2}\right) g_{n}(x) \leq k_{n}<\infty
$$


Then there exists an absolute constant $C>0$ such that

$$
\sup _{x}\left(1+x^{2}\right)\left|P\left[X_{1}+\ldots+X_{n} \leq x\right]-G_{n}(x)\right| \leq C k_{n}^{\frac{n}{n+1}}\left\{\max _{r=0,1,2} A_{X_{1} X_{2} \ldots X_{n}}^{r}\right\}^{\frac{1}{n+1}}
$$

where

$$
\begin{aligned}
& A_{X_{1} X_{2} \ldots X_{n}}^{r} \\
& \quad=\int_{-\infty}^{\infty} \ldots \int_{-\infty}^{\infty}\left|x_{1}+\ldots+x_{n}\right|^{r}\left|\operatorname{Cum}\left(\chi_{X_{1}}\left(x_{1}\right), \ldots, \chi_{X_{n}}\left(x_{n}\right)\right)\right| d x_{1} \ldots d x_{n}
\end{aligned}
$$

for $r=0,1,2$.

\section{Uniform and non-uniform bounds for functions of associated random variables}

Next we extend the results in Sections 2 and 3 to functions of associated random variables.

Let $g$ be a continuous function from $R^{n} \rightarrow R$ such that for any $x \in R^{n}$ and any $k=1, \ldots, n$ there exist finite dervatives $\frac{d^{+} g(x)}{d x_{k}}$ and $\frac{d^{-} g(x)}{d x_{k}}$. Further suppose that for each $k=1, \ldots, n$ there are atmost finite number of points $x$ at which $\frac{d^{+} g(x)}{d x_{k}} \neq \frac{d^{-} g(x)}{d x_{k}}$. Let

$$
L_{k}(g)=\max \left\{\left\|\frac{d^{+} g(x)}{d x_{k}}\right\|_{\infty},\left\|\frac{d^{-} g(x)}{d x_{k}}\right\|_{\infty}\right\}
$$

Lemma 4.1: Let $\left\{X_{n}, n \geq 1\right\}$ be a sequence of associated random variables. Let $g_{j}, j=$ $1, \ldots, n$ be functions defined as above. Then

$$
\begin{gathered}
\left|E\left[\exp \left(i t \sum_{j=1}^{n} g_{j}\left(X_{j}\right)\right)\right]-\prod_{j=1}^{n} E\left[\exp \left(i t g_{j}\left(X_{j}\right)\right)\right]\right| \\
\leq t^{2} \sum_{1 \leq j<k \leq n} L_{j}\left(g_{j}\right) L_{k}\left(g_{k}\right) \operatorname{Cov}\left(X_{j}, X_{k}\right) .
\end{gathered}
$$

Proof : We prove the result by induction. For $n=2$ using Newman's inequality

$$
\begin{aligned}
\mid E & {\left[\exp i t\left(g_{1}\left(X_{1}\right)+g_{2}\left(X_{2}\right)\right)\right]-\prod_{j=1}^{2} E\left[\exp i t g_{j}\left(X_{j}\right)\right] \mid } \\
& =\left|\operatorname{Cov}\left(\exp i t g_{1}\left(X_{1}\right), \exp i t g_{2}\left(X_{2}\right)\right)\right| \\
& \leq t^{2} L_{1}\left(g_{1}\right) L_{2}\left(g_{2}\right) \operatorname{Cov}\left(X_{1}, X_{2}\right) .
\end{aligned}
$$

Suppose that the result is true for $n=M$. Then for $n=M+1$, using Bulinski's inequality (1996) and the induction hypothesis we get

$$
\left|E\left[\exp i t \sum_{j=1}^{M+1} g_{j}\left(X_{j}\right)\right]-\prod_{j=1}^{M+1} E\left[\exp i t g_{j}\left(X_{j}\right)\right]\right|
$$




$$
\begin{aligned}
& =\mid \operatorname{Cov}\left(\exp i t \sum_{j=1}^{M} g_{j}\left(X_{j}\right), \exp i t g\left(X_{M+1}\right) \mid\right. \\
& +\left|E\left[\exp i t g\left(X_{M+1}\right)\right]\right|\left|E\left[\exp i t \sum_{j=1}^{M} g_{j}\left(X_{j}\right)\right]-\prod_{j=1}^{M} E\left[\exp i t g_{j}\left(X_{j}\right)\right]\right| \\
& \leq t^{2} \sum_{j=1}^{M} L_{j}\left(g_{j}\right) L_{M+1}\left(g_{M+1}\right) \operatorname{Cov}\left(X_{j}, X_{M+1}\right)+t^{2} \sum_{1 \leq j<k \leq M} L_{j}\left(g_{j}\right) L_{k}\left(g_{k}\right) \operatorname{Cov}\left(X_{j}, X_{k}\right) \\
& =t^{2} \sum_{1 \leq j<k \leq M+1} L_{j}\left(g_{j}\right) L_{k}\left(g_{k}\right) \operatorname{Cov}\left(X_{j}, X_{k}\right)
\end{aligned}
$$

which proves the result.

We use this lemma to prove uniform Berry-Esseen bounds for functions of associated random variables.

Theorem 4.2 : Let $\left\{X_{n}, n \geq 1\right\}$ be a sequence of stationary associated random variables. Let $g$ be a differentiable function with

$$
\sup _{x}\left|g^{\prime}(x)\right|<C
$$

Further suppose that $E\left[g\left(X_{1}\right)\right]=0, \operatorname{Var}\left[g\left(X_{1}\right)\right]=\sigma_{1}^{2}>0, E\left[\left|g\left(X_{1}\right)\right|^{3}\right]<\infty$ and $g\left(X_{1}\right)$ has an absolutely continuous distribution. Let $V_{n}=\sum_{i=1}^{n} g\left(X_{i}\right)$ and $v_{n}^{2}=\operatorname{Var} V_{n}$. Suppose $\frac{v_{n}^{2}}{n} \rightarrow \sigma_{1}^{2}$ as $n \rightarrow \infty$. Let $H_{n}(x)$ be the distribution function of $\frac{V_{n}}{v_{n}}$ and $H_{n}^{*}($.$) be the distribution function$ of $\frac{\sum_{i=1}^{n} Z_{i}^{*}}{v_{n}}$, where $Z_{i}^{*}, 1 \leq i<n$ are i.i.d with distribution function same as that of $g\left(X_{1}\right)$. Let $m_{n}^{*}$ be a bound on the density of $H_{n}^{*}$. Then there exist absolute constants $C_{i}>0,1 \leq i \leq 3$, such that

$$
\begin{aligned}
\sup _{x}\left|H_{n}(x)-\Phi(x)\right| & \leq C_{1} \sup _{x}\left(g^{\prime}(x)\right)^{2} \frac{d_{n}{ }^{1 / 3} m_{n}^{* 2 / 3}}{v_{n}{ }^{2 / 3}} \\
& +C_{2} \frac{E\left|g\left(X_{1}\right)\right|^{3}}{\sqrt{n} \sigma_{1}^{3}}+C_{3}\left(\frac{v_{n}}{\sigma_{1} \sqrt{n}}-1\right) .
\end{aligned}
$$

The proof follows on the same lines as Theorem 2.1 by using Lemma 4.1.

The following theorem gives the non-uniform bounds for functions of associated random variables. Note that $\beta_{1 n}=\int_{-\infty}^{\infty} x^{2} d H_{n}(x)=1$ and $\beta_{2 n}=\int_{-\infty}^{\infty} x^{2} d H_{n}^{*}(x)$ is bounded by the condition $\frac{v_{n}^{2}}{n} \rightarrow \sigma_{1}^{2}$ as $n \rightarrow \infty$.

Theorem 4.3 : Let $\left\{X_{n}, n \geq 1\right\}$ be a sequence of stationary associated random variables. Let $g$ be a differentiable function with

$$
\sup _{x}\left|g^{\prime}(x)\right|<\infty .
$$

Suppose the conditions of Theorem 4.2 hold. Then there exist absolute positive constants $C$ and $c(2)$ such that

$$
\left|H_{n}(x)-\Phi(x)\right| \leq C\left\{\frac{2 \Delta_{1 n}^{*}}{1+x^{2}}\right\}^{1 / 2}+\frac{c(2) \Delta_{2 n}^{*} \log \left(1 / \Delta_{2 n}^{*}\right)+\lambda_{2 n}^{*}}{1+x^{2}},
$$


for all $x$ where

$$
\begin{aligned}
\Delta_{1 n}^{*} & =\sup _{x}\left|H_{n}(x)-H_{n}^{*}(x)\right|, \\
\Delta_{2 n}^{*} & =\sup _{x}\left|H_{n}(x)-\Phi(x)\right|, \quad \text { and } \\
\lambda_{2 n}^{*} & =\left|\int_{-\infty}^{\infty} x^{2} d H_{n}^{*}(x)-1\right| \\
& =\left|n \sigma_{1}^{2} / v_{n}^{2}-1\right| .
\end{aligned}
$$

\section{Applications}

We consider an application of the above result to kernel type density estimation. We obtain a bound on the expected value of the integrated mean deviation of a kernel-type density estimator $f_{n}$ for the true density function $f$.

Let us first assume that $X_{1}, \ldots, X_{n}$ be i.i.d. random variables with the probability density function $f$. Let $f_{n}(x)$ be a kernel-type density estimator where $f_{n}(x)$ is given by

$$
f_{n}(x)=\frac{1}{n h_{n}} \sum_{i=1}^{n} K\left(\frac{x-X_{i}}{h_{n}}\right)
$$

where $K$ is a symmetric bounded differentiable density function with compact support. Further suppose that

$$
\begin{gathered}
\lim _{|u| \rightarrow \infty}|u| K(u)=0 \\
\int_{-\infty}^{\infty} u^{2} K(u) d u<\infty \\
\sup _{x} \mid K^{\prime}(x) \leq C
\end{gathered}
$$

where $h_{n}$ is a sequence of positive numbers satisfying

$$
\lim _{n \rightarrow \infty} h_{n}=0 \quad \lim _{n \rightarrow \infty} n h_{n}=\infty .
$$

Let $\mathcal{F}$ be the class of densities $f$ satisfying

(i) $f$ is absolutely continuous with a.e. dervative $f^{\prime}$;

(ii) $f^{\prime}$ is absolutely continuous with a.e. dervative $f^{\prime \prime}$ and

(iii) $f^{\prime \prime}$ is continuous and bounded.

Then Devroye and Gyorfi (1985) proved the following result

Theorem 5.1 : For all $f$ in $\mathcal{F}$ having compact support, the kernel estimate defined above satisfies

$$
E \int_{-\infty}^{\infty}\left|f_{n}(x)-f(x)\right| d x=J\left(n, h_{n}\right)+o\left(h_{n}^{2}+\left(n h_{n}\right)^{-1 / 2}\right),
$$

where

$$
J\left(n, h_{n}\right)=\int_{-\infty}^{\infty} \frac{\alpha \sqrt{f(x)}}{\sqrt{n h_{n}}} \psi\left(\sqrt{n h_{n}^{5}} \frac{\beta\left|f^{\prime \prime}(x)\right|}{2 \alpha \sqrt{f(x)}}\right) d x,
$$


where

$$
\psi(|a|)=|a| P(|N| \leq|a|)+\sqrt{\frac{2}{\pi}} e^{-\frac{a^{2}}{2}},
$$

and

$$
\alpha=\sqrt{\int_{-\infty}^{\infty} K^{2}(x) d x}, \quad \beta=\int_{-\infty}^{\infty} x^{2} K(x) d x .
$$

Here $N$ is a standard normal random variable.

We will extend the above result to a sequence of stationary associated random variables $\left\{X_{n}, n \geq 1\right\}$ using the non-uniform bound (3.10).

Lemma 5.2: Let $\left\{X_{n}, n \geq 1\right\}$ be a sequence of stationary associated random variables with common distribution function $F$ for $X_{1}$. Let the conditions of Theorem 2.1 hold. Then

$$
\begin{aligned}
& \sup _{a \in R}\left|E\left(\left|\frac{S_{n}}{s_{n}}-a\right|\right)-E(|N-a|)\right| \\
& \quad \leq C\left(\sqrt{\Delta_{1 n}}+\Delta_{2 n} \log \left(1 / \Delta_{2 n}\right)+\lambda_{2 n}\right)
\end{aligned}
$$

where $C$ is a universal positive constant and $\Delta_{1 n}, \Delta_{2 n}$ and $\lambda_{2 n}$ are as defined in Section 3.

Proof : Let $F_{n}$ be the distribution function of $\frac{S_{n}}{s_{n}}$. Let $\Phi$ be the distribution function of a standard normal random variable.

Then

$$
\begin{aligned}
E\left(\left|\frac{S_{n}}{s_{n}}-a\right|\right) & =\int_{0}^{\infty} P\left(\left|\frac{S_{n}}{s_{n}}-a\right|>t\right) d t \\
& =\int_{0}^{\infty}\left(1-F_{n}(a+t)+F_{n}(a-t)\right) d t
\end{aligned}
$$

Similarly

$$
E(|N-a|)=\int_{0}^{\infty}(1-\Phi(a+t)+\Phi(a-t)) d t
$$

Therefore

$$
\left.\left|E\left(\left|\frac{S_{n}}{s_{n}}-a\right|\right)-E(|N-a|)\right|\right\} \leq \int_{-\infty}^{\infty}\left|\Phi(t)-F_{n}(t)\right| d t .
$$

The result now follows from integrating the inequality in (3.10) and using the facts

$$
\sqrt{1+x^{2}} \leq\left(1+x^{2}\right)
$$

and

$$
\int_{-\infty}^{\infty} \frac{1}{\sqrt{1+x^{2}}} d x<c
$$

On similar lines, we can prove the following Lemma.

Lemma 5.3 : Let $\left\{X_{n}, n \geq 1\right\}$ be a sequence of stationary associated random variables with distribution function $F$ for $X_{1}$. Let $g$ be a differentiable function with $\sup _{x}\left|g^{\prime}(x)\right|<C$. Let $E\left(g\left(X_{1}\right)\right)=0, \operatorname{Var}\left(g\left(X_{1}\right)\right)=\sigma_{1}^{2}>0$, and $E\left[\left|g\left(X_{1}\right)\right|^{3}\right]<\infty$ and suppose that $g\left(X_{1}\right)$ has an 
absolutely continuous distribution function. Let $V_{n}=\sum_{i=1}^{n} g\left(X_{i}\right)$ and $v_{n}^{2}=\operatorname{Var} V_{n}$. Suppose $\frac{v_{n}^{2}}{n \sigma_{1}^{2}} \rightarrow 1$ as $n \rightarrow \infty$. Then

$$
\begin{aligned}
& \sup _{a \in R}\left|E\left(\left|\frac{V_{n}}{v_{n}}-a\right|\right)-E(|N-a|)\right| \\
& \leq C\left(\sqrt{\Delta_{1 n}^{*}}+\Delta_{2 n}^{*} \log \left(1 / \Delta_{2 n}^{*}\right)+\lambda_{2 n}^{*}\right)
\end{aligned}
$$

where $C$ is a positive constant and $\Delta_{1 n}^{*}, \Delta_{2 n}^{*}$ and $\lambda_{2 n}^{*}$ are as defined in (4.7).

Let $T$ be an arbitrary bounded interval. Let $K$ be a symmetric bounded differentiable density function with compact support. Suppose $[-r, r]$ is the support of the kernel $K$ and let $K^{*}$ be an upper bound for $K$. Suppose $\left\{X_{n}, n \geq 1\right\}$ is a stationary associated sequence of random variables with density function $f$ for $X_{1}$. Let $f_{n}(x)$, as given by (5.1) be a kernel estimator of $f(x)$.

Lemma 5.4 : Under the conditions stated above, for any $x$,

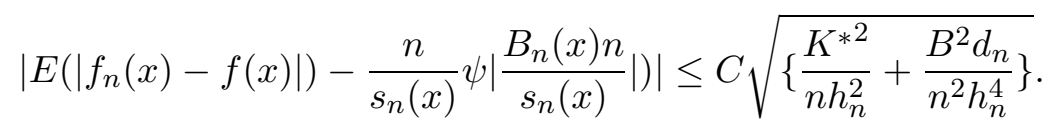

Proof : Let

$$
Y_{i}=g\left(X_{i}\right)=\frac{1}{h_{n}} K\left(\frac{x-X_{i}}{h_{n}}\right)-E \frac{1}{h_{n}} K\left(\frac{x-X_{i}}{h_{n}}\right)
$$

where $g($.$) satisfies the conditions in Lemma 5.3. Then$

$$
f_{n}(x)=\frac{1}{n} \sum_{i=1}^{n} Y_{i}=\frac{S_{n}}{n}
$$

and

$$
E S_{n}=0, s_{n}^{2}(x)=\operatorname{Var}\left(S_{n}\right), \quad \sigma_{n}^{2}(x)=\operatorname{Var} f_{n}(x)=\frac{s_{n}^{2}(x)}{n^{2}}
$$

Note that

$$
B_{n}(x)=E\left(f_{n}(x)\right)-f(x) .
$$

Then, choosing $a=-\frac{B_{n}(x) n}{s_{n}(x)}$, in Lemma 5.3 we get

$$
\begin{aligned}
& \left.|E| \frac{\sum_{i=1}^{n} Y_{i}}{\operatorname{Var}\left(\sum_{i=1}^{n} Y_{i}\right.}\right)-a|-\psi(|a|)| \\
& \quad=|E| \frac{f_{n}(x)-E f_{n}(x)}{\sqrt{\operatorname{Var} f_{n}(x)}}-a|-\psi(|a|)| \\
& \quad=|E| \frac{f_{n}(x)-f(x)}{\sqrt{\operatorname{Var} f_{n}(x)}}-\frac{B_{n}(x)}{\sqrt{\operatorname{Var} f_{n}(x)}}-a|-\psi(|a|)| \\
& \quad=\frac{1}{\sqrt{\operatorname{Var} f_{n}(x)}}|E| f_{n}(x)-f(x)\left|-\frac{n}{s_{n}(x)} \psi\left(\left|\frac{B_{n}(x) n}{s_{n}(x)}\right|\right)\right| \\
& \quad \leq C\left(\sqrt{\Delta_{1 n}^{*}}+\Delta_{2 n}^{*} \log \left(1 / \Delta_{2 n}^{*}\right)+\lambda_{2 n}^{*}\right) .
\end{aligned}
$$


Hence

$$
\begin{aligned}
& |E| f_{n}(x)-f(x)\left|-\frac{n}{s_{n}(x)} \psi\left(\left|\frac{B_{n}(x) n}{s_{n}(x)}\right|\right)\right| \\
& \quad \leq c \sqrt{\operatorname{Var} f_{n}(x)}\left(\sqrt{\Delta_{1 n}^{*}}+\Delta_{2 n}^{*} \log \left(1 / \Delta_{2 n}^{*}\right)+\lambda_{2 n}^{*}\right) .
\end{aligned}
$$

Since $\frac{v_{n}^{2}}{n \sigma_{1}^{2}} \rightarrow 1$, we get

$$
\lambda_{2 n}^{*} \rightarrow 0 \text { as } n \rightarrow \infty
$$

From the Newman's inequality we get that

$$
\left|\operatorname{Cov}\left(K\left(\frac{x-X_{i}}{h_{n}}\right), K\left(\frac{x-X_{j}}{h_{n}}\right)\right)\right| \leq \frac{B^{2}}{h_{n}^{2}} .
$$

Further note that

$$
\begin{aligned}
\operatorname{Var} f_{n}(x) & =\frac{1}{n h_{n}^{2}} \operatorname{Var} K\left(\frac{x-X_{1}}{h_{n}}\right)+\frac{1}{n^{2} h_{n}^{2}} \sum_{1 \leq i \neq j \leq n} \operatorname{Cov}\left(K\left(\frac{x-X_{i}}{h_{n}}\right), K\left(\frac{x-X_{j}}{h_{n}}\right) \mid\right. \\
& \leq \frac{K^{* 2}}{n h_{n}^{2}}+\frac{B^{2} d_{n}}{n^{2} h_{n}^{4}} .
\end{aligned}
$$

Since $\Delta_{1 n}^{*}, \Delta_{2 n}^{*}$ and $\lambda_{2 n}^{*} \rightarrow 0$ as $n \rightarrow \infty$, the result follows from (5.16) and (5.22)

Remark : The condition $\frac{s_{n}^{2}}{n \sigma_{1}^{2}} \rightarrow 1$ holds under the condition (A1) (iv) discussed by Roussas (2000).

Lemma 5.5 : Let $T$ be a bounded interval . Suppose that

$$
\frac{1}{n h_{n}^{4}} \sum_{j=1}^{n} \operatorname{Cov}\left(X_{1}, X_{j}\right) \rightarrow 0 \text { as } n \rightarrow \infty .
$$

Then, as $h_{n} \rightarrow 0$,

$$
\int_{T}\left|\frac{\sigma_{n}(x) \sqrt{n h_{n}}}{\alpha}-\sqrt{f(x)}\right| d x=o(1)
$$

where $\alpha$ is as defined in (5.9).

Proof: For all bounded sets $T$ we have $\int_{T} \sqrt{f(x)} d x<\infty$. Let $\lambda($.) denote the Lebesgue measure. Let

$$
\begin{gathered}
K_{h}(x)=K\left(\frac{x}{h}\right), \quad \tilde{K}(x)=\frac{K^{2}(x)}{\alpha^{2}} \\
f * K(x)=\int_{-\infty}^{\infty} f(y) K(x-y) d y=\int_{-\infty}^{\infty} K(y) f(x-y) d y
\end{gathered}
$$

Now

$$
\sigma_{n}^{2}(x)=a_{n}(x)+b_{n}(x)+c_{n}(x)
$$

where

$$
\begin{aligned}
a_{n}(x) & =\frac{\alpha^{2} f(x)}{n h_{n}} \\
b_{n}(x) & =\frac{\left(f * \tilde{K}_{h}(x)-f(x)\right) \alpha^{2}}{n h_{n}}-\frac{\left(f * K_{h}(x)\right)^{2}}{n} \\
c_{n}(x) & =\frac{1}{n^{2} h_{n}^{2}} \sum_{1 \leq i \neq j \leq n} \operatorname{Cov}\left(K\left(\frac{x-X_{i}}{h_{n}}\right), K\left(\frac{x-X_{j}}{h_{n}}\right)\right)
\end{aligned}
$$


Note that $a_{n}(x) \geq 0$. Let $b_{n}^{+}(x)$ and $c_{n}^{+}(x)$ denote the positive parts of $b_{n}(x)$ and $c_{n}(x)$. Note that

$$
\sqrt{a_{n}(x)+b_{n}(x)+c_{n}(x)} \leq \sqrt{a_{n}(x)}+\sqrt{b_{n}^{+}(x)}+\sqrt{c_{n}^{+}(x)} .
$$

Integrating over $T$ and applying the Cauchy-Schwarz inequality gives

$$
\begin{aligned}
\int_{T} \sigma_{n}(x) d x & \leq \frac{\alpha}{\sqrt{n h_{n}}}\left(\int_{T} \sqrt{f(x)} d x+\int_{T} \sqrt{\left|f * \tilde{K}_{h}(x)-f(x)\right|} d x\right)+\int_{T} \sqrt{c_{n}^{+}(x)} d x \\
& \leq \frac{\alpha}{\sqrt{n h_{n}}}\left(\int_{T} \sqrt{f(x)} d x+\sqrt{\int_{T}\left|f * \tilde{K}_{h}(x)-f(x)\right| d x \lambda(T)}\right)+\sqrt{\int_{T}\left|c_{n}(x)\right| d x \lambda(T)}
\end{aligned}
$$

Further,

$$
\sqrt{a_{n}(x)+b_{n}(x)+c_{n}(x)} \geq \sqrt{a_{n}(x)}-\sqrt{\left|b_{n}(x)\right|}-\sqrt{\left|c_{n}(x)\right|} .
$$

Therefore,

$$
\begin{aligned}
\int_{T} \sigma_{n}(x) d x \geq & \frac{\alpha}{\sqrt{n h_{n}}}\left(\int_{T} \sqrt{f(x)} d x-\sqrt{\int_{T}\left|f * \tilde{K}_{h}(x)-f(x)\right| d x \lambda(T)}\right. \\
& \left.-\frac{\sqrt{h_{n}}}{\alpha} \int_{T} f * K_{h}(x) d x\right)-\sqrt{\int_{T}\left|c_{n}(x)\right| d x \lambda(T)} .
\end{aligned}
$$

Using the stationarity of the sequence $\left\{X_{n}\right\}$ and Bulinski's inequality, it is easy to see that

$$
\left|c_{n}(x)\right| \leq \frac{C}{n h_{n}^{4}} \sum_{j=1}^{n} \operatorname{Cov}\left(X_{1}, X_{j}\right) .
$$

Furthermore $\lambda(T)<\infty$ and

$$
\lim _{h_{n} \rightarrow 0} \int_{-\infty}^{\infty}\left|f * K_{h}(x)-f(x)\right| d x=0 .
$$

The result now follows by combining (5.27), (5.29) to (5.31).

Remark 5.6 : Condition (5.21) will hold if $\operatorname{Cov}\left(X_{1}, X_{j}\right)=j^{-r}, 4 / 5<r<1$ and $h_{n}=n^{-\frac{1}{5}}$.

For proof of the following two Lemmas, see Devroye and Gyorfi (1985).

Lemma 5.7 : For all $f \in \mathcal{F}$ and for all symmetric bounded density functions $K$ with compact support and all bounded intervals $T$,

$$
\int_{T}\left|B_{n}(x)-\frac{\beta}{2} h_{n}^{2}\right| f^{\prime \prime}(x)|| d x=o\left(h_{n}^{2}\right) \text { as } h_{n} \rightarrow 0 .
$$

The same remains true if the set $T$ is replaced by $R$ whenever $f$ has compact support $f \in \mathcal{F}$, and $K$ is a bounded symmetric density function with compact support.

Lemma 5.8 : For nonnegative numbers $u, v, w, z$, we have

$$
\left|u \psi\left(\frac{v}{u}\right)-w \psi\left(\frac{z}{w}\right)\right| \leq|v-z|+\sqrt{\frac{2}{\pi}}|u-w|
$$


where $\psi$ is as defined in (5.8).

Theorem 5.9 : Let $\left\{X_{n}\right\}$ be a sequence of stationary associated random variables with $f$ as the density function of $X_{1}$. Let $f \in \mathcal{F}$ have compact support. Let $f_{n}(x)$ be the kernel type density estimator given by (5.1). Let the conditions (5.2), (5.5) and (5.21) hold. Then

$$
E \int_{-\infty}^{\infty}\left|f_{n}(x)-f(x)\right| d x=J\left(n, h_{n}\right)+o\left(h_{n}^{2}+\left(n h_{n}\right)^{-1 / 2}\right)+o\left(\frac{\sqrt{d_{n}}}{n h_{n}^{2}}\right),
$$

where

$$
J\left(n, h_{n}\right)=\int_{-\infty}^{\infty} \frac{\alpha \sqrt{f(x)}}{\sqrt{n h_{n}}} \psi\left(\sqrt{n h_{n}^{5}} \frac{\beta\left|f^{\prime \prime}(x)\right|}{2 \alpha \sqrt{f(x)}}\right) d x,
$$

where $\psi(|a|), \alpha$ and $\beta$ are as defined in (5.8) and (5.9).

Proof : First suppose that $f \in \mathcal{F}$ and $f$ has compact support in a bounded interval $T$. Take $T$ so large that for every $x$ in the support of $f$, the interval $[x-a, x+a]$ is contained in $T$, where $a$ is a number sufficiently large so that $K_{h_{n}}(u)=0$ for all $n$ and all $|u|>a$.

Take

$$
\begin{gathered}
u=\frac{s_{n}(x)}{n} ; \quad v=\left|B_{n}(x)\right| \\
w=\frac{\alpha \sqrt{f(x)}}{\sqrt{n h_{n}}} ; \quad z=\frac{\beta}{2} h_{n}^{2}\left|f^{\prime \prime}(x)\right|
\end{gathered}
$$

in Lemma 5.8.

From Lemmas 5.5 and 5.7, we get that

$$
\int_{T}|v-z| d x=o\left(h_{n}^{2}\right)
$$

and

$$
\int_{T}|u-w| d x=o\left(\left(n h_{n}\right)^{-\frac{1}{2}}\right) .
$$

Combining it with Lemma 5.3, we get

$$
\begin{aligned}
& \left|\int_{T} E\left(\left|f_{n}(x)-f(x)\right|\right) d x-J\left(n, h_{n}\right)\right| \\
& \quad \leq\left|\int_{T} E\left(\left|f_{n}(x)-f(x)\right|\right) d x-\int_{T} \frac{s_{n}(x)}{n} \psi\left(\frac{n\left|B_{n}(x)\right|}{s_{n}(x)}\right)\right| d x \\
& \quad+\left|\int_{T} \frac{s_{n}(x)}{n} \psi\left(\frac{n\left|B_{n}(x)\right|}{s_{n}(x)}\right) d x-J\left(n, h_{n}\right)\right| \\
& \quad \leq \sqrt{\frac{K^{* 2}}{n h_{n}}+\frac{B^{2} d_{n}}{n^{2} h_{n}^{4}}}+o\left(h_{n}^{2}\right)+o\left(\left(n h_{n}\right)^{-\frac{1}{2}}\right)
\end{aligned}
$$

where $J\left(n, h_{n}\right)=\int_{T} w \psi(z / w) d x$.

This proves the result. 


\section{REFERENCES}

1. Bagai, I and Prakasa Rao, B.L.S. (1991) Estimation of the survival function for stationary associated processes, Statist. Probab. Lett. 12, 385-391.

2. Bagai, I. and Prakasa Rao, B.L.S. (1995) Kernel-type density and failure rate estimation for associated sequences, Ann. Inst. Statist. Math. 47, 253-266.

3. Birkel, T. (1988) On the convergence rate in the central limit theorem for associated processes, Ann. Probab. 16, 1685-1693.

4. Block, H.W. and Fang, Z. (1988) A multivariate extension of Hoeffding's lemma.Ann. Probab., 16, 1803-1820.

5. Bulinski, A.V. (1996) On the convergence rates in the CLT for positively and negatively dependent random fields. pp 1-14. In Probability Theory and Mathematical Statistics ., eds, Ibragimov, I.A. and Zaitsev, A.Yu, Gordon and Breach, Amsterdam.

6. Devroye, L. and Gyorfi, L. (1985) Nonparametric Density Estimation - The L $L_{1}$ view. John Wiley, New York.

7. Dewan, I. and Prakasa Rao, B.L.S. (1997) Remarks on Berry-Esseen type bound for stationary associated random variables, Gujarat Statist. Rev. , 24, 19-20.

8. Dewan, I. and Prakasa Rao, B.L.S. (2000) Explicit bounds on Levy-Prohov distance for a class of multidimensional distribution functions, Statist. Probab. Letters , 48, 105-119.

9. Esary, J, Proschan, F. and Walkup, D. (1967) Association of random variables with applications, Ann. Math. Statist. 38, 1466-1474.

10. Esseen, C.G. (1956) A moment inequality with an application to the central limit theorem, Skand. Aktuarie. 39, 160-170.

11. Feller, W. (1977) An Introduction to Probability Theory and Its Applications, Vol II, Wiley Eastern, New Delhi.

12. Hall, P. (1982) Rates of convergence in the central limit theorem, Pitman, Boston.

13. Newman, C.M. and Wright, A.L. (1981) An invariance principle for certain dependent sequences, Ann. Probab., 9, 671-675.

14. Petrov, V.V. (1975) Sums of Independent Random Variables, Springer - Verlag, New York.

15. Prakasa Rao. B.L.S. and Dewan, I. (2001) Associated sequences and related inference problems, Handbook of Statistics, 19 , Stochastic Processes: Theory and Methods, (eds. C.R. Rao and D.N. Shanbag), 693-728, North Holland, Amsterdam. 
16. Roussas, G.G. (1999) Positive and negative dependence with some statistical applications, Asymptotics, nonparametrics and time series (ed. S. Ghosh), 757-788, Marcel Dekker, New York.

17. Roussas, G.G. (2000) Asymptotic normality of the kernel estimate of a probability density function under association, Statist. Probab. Letters, 50, 1-12.

18. Van Beek, P. (1972) An application of Fourier methods to the problem of sharpening the Berry-Esseen inequality, Z. Wahrscheinlichkeitstheorie Verw.Geb. , 23, 187-196.

19. Wood, T.E. (1983) A Berry-Esseen theorem for associated random variables, Ann. Probab., 11, 1042-1047. 